\title{
An Alternating Quark Sequence Subnucleonic Structure of Stable Light Nuclei H-1 Through Li-7
}

\author{
Raymond J. Walsh* \\ Keywords: AQS, alternating quark sequence, quark structure, subnucleonic structure, nuclear structure, light nuclei, RMS charge radius, nuclear \\ radius, model of the atomic nucleus, AQS parameters, quark geometry, ball and stick, spherical nucleus, close packed, close packing, proton puzzle, \\ nucleosynthesis, harmonic oscillator.
}

\begin{abstract}
Structures of stable light nuclei incorporate an alternating up and down quark sequence (AQS) of equally spaced quarks around regular polyhedron geometries. AQS is a ball-and-stick model in which the ball represents the average center of current quark mass, and the stick length is constant and equal to the radius of the proton. AQS radius predictions of $\mathrm{H}-1$ through Li-7 demonstrate 99.3\% average agreement (SD 4\%) and statistical correlation of $\rho=0.96, \mathrm{p}<0.001$, with accepted RMS charge radii. Light nuclei above deuterium conform to ring structures whose radii are calculated from the formula of a regular polygon having $n$ sides, each side equal to the radius of the proton, and $n$ vertices, each occupied by a quark. Quark-quark interactions link nucleons to maintain a continuous sequence of alternating equally spaced quarks. Parallel strands of quark sequences overlap so that protons overlap with neutrons. Regular polyhedron structures yield better radius predictions; larger nuclei tend to be less regular and less predictable (with the exception of C-12). The relative certainty in the accepted radius of helium-4, and its geometric relationship tithe proton radius, allow a prediction for the proton radius of $0.8673 \pm 0.0014 \mathrm{fm}$. ASQ localizes average quark position, and the high statistical correlation of ASQ with experimental radii thus warrants an expression of the uncertainty principle as the product of uncertainty in energy and time rather than position and momentum. This view is consistent with the quark as a harmonic oscillator.
\end{abstract}

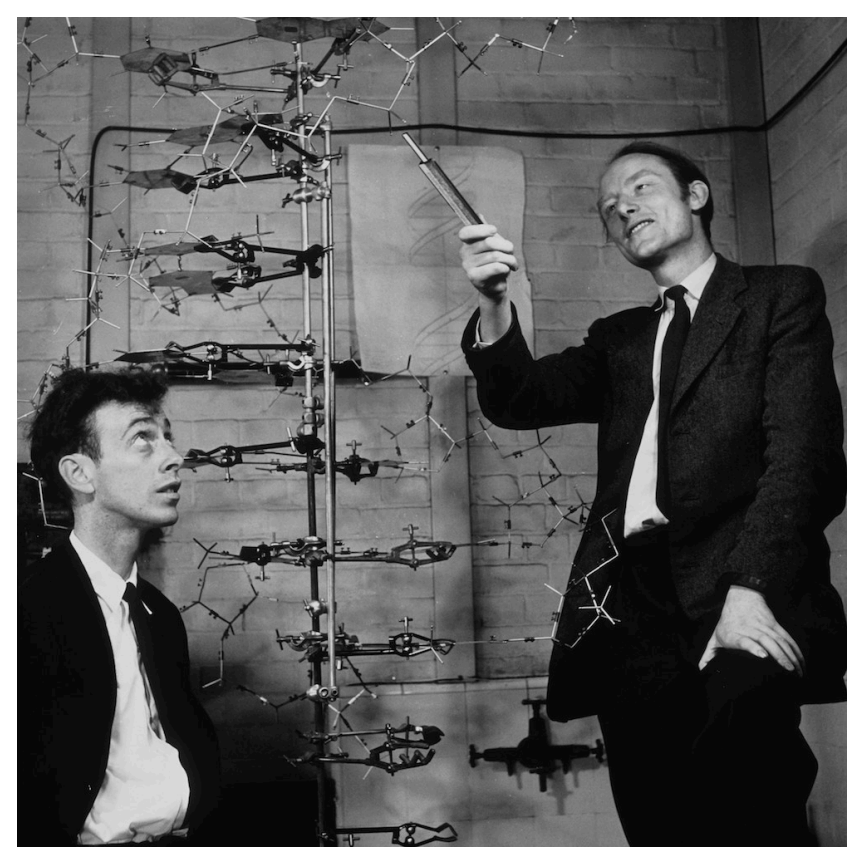

Figure 1. Molecular models represent the relative positions of atoms and are useful for communicating structure. Watson and Crick used the ball-and-stick molecular model shown above to elucidate the structure of DNA. Photo credit A. Barrington Brown, Science Photo Library.

\section{INTRODUGTION}

The fine tradition of the Ball-and-Stick Model dates back a century and a half, ${ }^{1}$ and today no chemistry classroom is complete without it. Though crude and simplistic, it has been an invaluable aid in helping chemists communicate molecular structure. Watson and Crick famously used ball-and-stick models to work out the structure of the double helix, including its specific ratio of base pairs and the unique x-ray crystallographic pattern. ${ }^{2}$ Black and white photos from the era show the DNA model winding above the jocular pair, a testament to their towering achievement.

Traditional ball-and-stick molecular models, such as those used by Watson and Crick, are representational and are useful for conveying the relative positions of atoms within a molecular structure. The first molecular model was unveiled by von Hofmann in 1861 to illustrate the relative positions of hydrogen and carbon in a molecule of methane. ${ }^{1}$ This model appears in the history of chemistry over a half century before Lewis dot structures would indicate a role for electron pairs in the bonding of atoms, ${ }^{3}$ and three quarters of a century before Linus Pauling would explain the quantum mechanical attractive forces holding these atoms in relative position. ${ }^{4}$ Von Hofmann demonstrated that purely structural or positional information can be useful, and positional information was certainly instrumental in leading Watson and Crick to the structure of DNA.

Running parallel to the elucidation of DNA, scientists were refining methods of probing the size and nature of the atomic nucleus. Ernest Rutherford discovered the nucleus by focusing 
alpha particles at a thin sheet of gold foil. ${ }^{5}$ Beams of electrons were later used instead of alpha particles to further define the nucleus. This process, known as deep inelastic scattering (DIS), not only revealed the size and shape of nuclei but also uncovered the existence of quarks and their strange fractional charges: $+2 / 3$ for the up quark and $-\mathbf{1} / \mathbf{3}$ for the down quark. Each nucleon has 3 quarks: two up and one down for the proton yields a charge of +1 , one up and two down for the neutron yields a charge of 0 .

The discovery of the quark sparked an interest in quark models of the atomic nucleus, but none have entered the main stream. As the late Norman Cook puts it: "In principle, a rigorous quark (parton) theory should underlie all of nuclear physics and eventually allow for the deduction of the relatively macroscopic properties of the nucleus on the basis of a more microscopic particle theory. Since the late 1970s, various models of the quark contribution to nuclear physics have been suggested ...but have not yet had a major influence on the traditional issues of nuclear structure theory." 6 While awaiting consensus on a comprehensive theoretical model, a limited representational model based on an alternating and equally spaced sequence of up and down quarks (AQS) within simple regular polyhedra appears consistent with experimental RMS charge radii.

The scope of this paper is limited to the elements of greatest interest to nuclear fusion theory, including the seven stable isotopes of hydrogen, helium, and lithium. Progress in the emerging field of low energy nuclear reactions (LENR) has been inhibited by lack of a viable theory, ${ }^{7}$ and a viable structural model might be helpful. In hot fusion the role of kinetic energy is central to overcoming the Coulomb barrier. ${ }^{8} \mathrm{~A}$ three-dimensional nuclear structure might reveal theoretical roles for rotational and vibrational kinetic energy in addition to the implied role of translational kinetic energy.

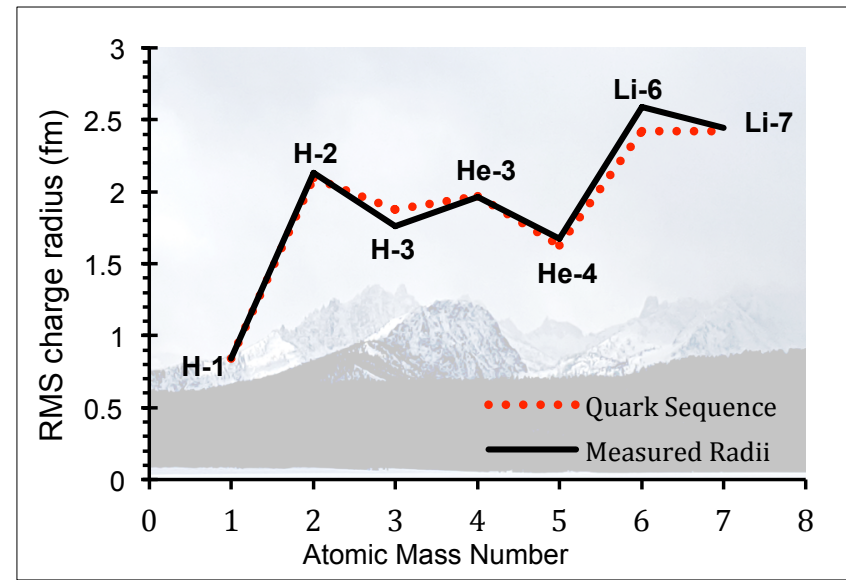

Figure 2. Correlation of the Alternating Quark Sequence model (red dots) with the measured root mean squared (RMS) charge radii of the first seven stable nuclides (black line). The plot resembles the Sawtooth Mountains of Central Idaho (background photo credit the author).

In general, the nuclear radius increases with mass number in predictable curvilinear fashion for elements above lithium-7. The graph of lighter elements, however, resembles the Sawtooth Mountains of Central Idaho (Figure 2). An alternating sequence of equally spaced quarks (AQS) can account for this saw tooth profile using a set of ball-and-stick models. The ball will represent the center of quark mass and the stick length is constant and equal to the radius of the proton. As a predictor of size and structure, AQS compares favorably with two other familiar representational models of the nucleus, namely closepacked protons and neutrons, and the more simplistic nucleus as a single sphere.

\section{- RESULTS AND DISGUSSION}

A central premise of AQS is that quark number determines nuclear structure. The number of up and down quarks within each nuclide is known from the number of protons and neutrons. The goal of AQS is to arrange the quarks of each nuclide into simple geometric shapes that best account for the measured RMS charge radius of each nuclide. A set of parameters for constructing AQS ball and stick models will follow.

Consider the first two stable nuclides. The proton, H-1, has an RMS charge radius $r=0.84 \mathrm{fm} .{ }^{9}$ The deuteron, H-2, has both a proton and a neutron. The two nucleons are roughly the same size so one might expect the deuteron to have a radius double that of proton $(2 \times 0.84=1.68 \mathrm{fm})$. In fact, the measured RMS charge radius of the deuteron is much larger: $r=2.13 \mathrm{fm} .^{9}$

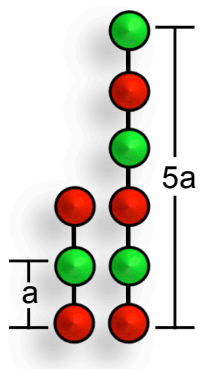

H-1 H-2
This numeric puzzle is more easily solved with a diagram. Red circles are up quarks and green circles are down quarks. The distance $a$ is the distance between adjacent quarks (in this and subsequent graphics). The radius of the proton is half the distance between the two up quarks on either end of $\mathrm{H}$ 1. In this model, the center of the proton corresponds to its central down quark. Thus the interquark distance $a$ is equal to the radius of the proton $(0.8414 \mathrm{fm})$.

Within the AQS model, the distance $a$ is the distance between adjacent quarks in the deuteron. From the picture it is evident that the diameter of $\mathrm{H}-2$ is 5 times the radius of $\mathrm{H}-1$, and thus the radius of $\mathrm{H}-2$ is $2 \frac{1}{2}$ times the radius of $\mathrm{H}-1$. The product of $2 \frac{1}{2} \times 0.84 \mathrm{fm}$ is $2.10 \mathrm{fm}$, representing $99 \%$ agreement with the experimental RMS charge radius of deuteron $(2.13 \mathrm{fm})$. AQS is able to reconcile the anomalous size difference between the proton and deuteron only if 1.) Quarks are arranged sequentially, and 2.) Adjacent quarks are spaced

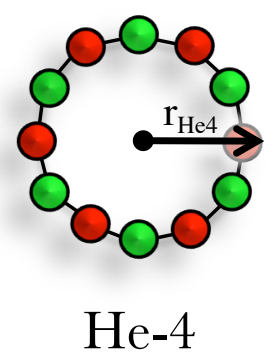
$0.8414 \mathrm{fm}$ apart. These are the first two parameters of AQS, and they hold for the other nuclides as well.

Helium-4 has an experimental radius of $1.68 \mathrm{fm} .{ }^{10} \mathrm{He}-4$ has twice the number of nucleons as $\mathrm{H}-2$ but is significantly smaller than $\mathrm{H}-2$, which has an experimental radius of $2.13 \mathrm{fm}$. To account for this size discrepancy, the 12 quarks of He-4 are arranged according to AQS in a regular dodecagon (12-gon) having sides $a=0.8414 \mathrm{fm}$ (equivalent to the radius of the proton). The black arrow represents the radius $r$, and the black dot at the origin of the arrow represents the average center of mass in this and subsequent drawings.

The helium- 4 radius $r$ is related to the interquark distance $a$ according to the regular polygon circumradius formula: 
(1)

$$
\mathrm{r}=\frac{a}{2} \csc \left(\frac{\pi}{n}\right)
$$

The value of $n$ equals the number of sides in the polygon, in this case 12. Solving for $\mathbf{r}$ yields an ASQ predicted radius of He-4 of $1.63 \mathrm{fm}$, representing $97 \%$ agreement with the experimental He-4 radius of $1.68 \mathrm{fm}$.

The AQS ring structure of He-4 is consistent with modelindependent analysis by J.S. McCarthy, et al, of electron scattering data that shows a significant central depression or hole in the nuclear matter distribution of $\mathrm{He}-4$ (and $\mathrm{He}-3$ as well) within a radius $0.8 \mathrm{fm} . .^{11}$

The dodecagon structure of helium-4 illustrates a third

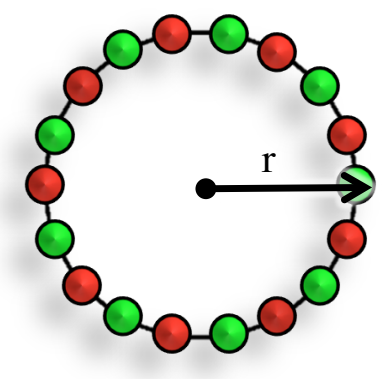

Li-6 AQS parameter: Since the vertex angle of a dodecagon is $150^{\circ}$, and there are no smaller circular structures in the AQS model, the lower limit of flexure of three sequential quarks is approximately $150^{\circ}$. (In the AQS Corrections section below it will be shown that this limit may be as low as $145.4^{\circ}$.)

This angle may manifest certain physical properties of the He-4 nucleus. The dodecagon ring has a relatively high number of quarks per unit volume compared to heavier nuclides, and may explain the relatively high nucleon density of He-4. Additionally, the acute vertex angle may result in ring strain that manifests in higher potential energy compared to the more lax ring structures below. This additional potential energy may explain the higher nucleon binding energy of He-4 compared to other light nuclei.

In similar fashion, the 18 quarks of Lithium-6 $(2.59 \mathrm{fm}){ }^{10}$ form a regular octadecagon (18-gon). The lithium-6 radius $r$ is related to the interquark distance $a$ according to formula (1), with $n=18$. The calculation yields an AQS predicted radius for Li-6 $2.42 \mathrm{fm}$, representing 95\% agreement with experimental Li- 6 radius of $2.59 \mathrm{fm}$. (This value improves with correction as shown below).

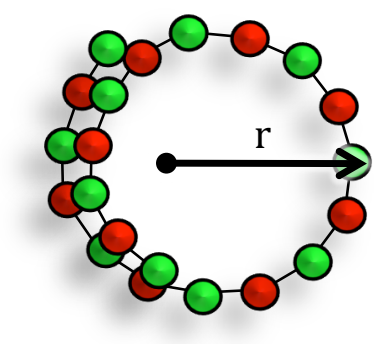

Li-7
The measured RMS charge radius of $\mathrm{Li}-7(2.44 \mathrm{fm}){ }^{10}$ is smaller than Li-6 (2.59 fm). The experimental value of $\mathrm{Li}-7$ fits with a pentadecagon (15-gon) structure with six quarks overlapping (aka a split ring). The lithium-7 radius $r$ is related to the interquark distance $a$ according to formula (1) with $n=15$. This yields a $\mathrm{r}=1.98 \mathrm{fm}$ for the 15-gon, woefully short of the experimentally determined RMS charge radius of $2.44 \mathrm{fm}$ for lithium-7.

The overlapping ends, however, shift the average center of mass $(\mathrm{cm})$ towards the overlapping strands and away from the geometric center of the 15-gon according to the standard three-dimensional center of mass formula:

$x_{c m}=\left(x_{1} m_{1}+x_{2} m_{2} \ldots+x_{n} m_{n}\right) /\left(m_{1}+m_{2} \ldots+m_{n}\right)$

(2) $y_{c m}=\left(y_{1} m_{1}+y_{2} m_{2} \ldots+y_{n} m_{n}\right) /\left(m_{1}+m_{2} \ldots+m_{n}\right)$

$z_{c m}=\left(z_{1} m_{1}+z_{2} m_{2} \ldots+z_{n} m_{n}\right) /\left(m_{1}+m_{2} \ldots+m_{n}\right)$ where points $\left(x_{n}, y_{n}, z_{n}\right)$ represent the Cartesian coordinates of each quark, and $m_{n}$ represents quark mass. Current quark masses are $m_{\mathbf{u}}=2.16 \mathrm{MeV}$ and $m_{\mathbf{d}}=4.67 \mathrm{MeV} .{ }^{12}$

The distance between overlapping strands is assumed to be $0.898 \mathrm{fm}^{13}$, the average distance between the nucleons within stable isotopes according to the International Data Committee. ${ }^{\text {T }}$ The calculated average center of mass, including $\mathrm{x}, \mathrm{y}$, and $\mathrm{z}$ components, is $2.42 \mathrm{fm}$ from the farthest quark on the opposite side. This prediction is within $1 \%$ of the measured RMS charge radius of $2.44 \mathrm{fm}$.

The ring structures of helium-4, lithium- 6 and lithium 7 illustrate additional parameters: 4.) up and down quarks alternate, 5.) nucleons are linked by opposite quarks to maintain a continous quark sequence, and 6.) quark strands may link indirectly or overlap in such a way that opposite quarks align and protons overlap with neutrons, and 7.) quark sequences tend to form ring structures.

He- 4 and Li-6 form closed ring structures with paired up and down quarks. This ring structure also requires that nucleons also pair: He-4 pairs two protons with two neutrons, while Li-6 pairs three protons with three neutrons.

The overlapping quark strands of Li-7 suggest that closed ring structures may not form when the number of up quarks and down quarks are unequal within the proposed ring, perhaps because like quarks might not be able to link. Any quark ring structure having an odd number of quarks would require at some point that a down quark link with a down quark, or an up quark with an up quark. If like quarks cannot link then ring structures must have an equal number of quarks. The equal quark requirement implies that the ring would also have an equal number of protons and neutrons.

The accepted RMS charge radii for $\mathrm{H}-3$ and He-3 are $1.76 \mathrm{fm}$ and $1.97 \mathrm{fm}$ respectively. ${ }^{10}$ These values are greater than He-4 (1.68 fm) but less than H-2 (2.13 fm). A linear structure for the nine quarks of $\mathrm{H}-3$ and $\mathrm{He}-3$ would result in excessively large radius predictions for each, but a circular arrangement (a nonagon) yields a circumradius of $1.23 \mathrm{fm}$, which is problematically small. A closer estimate of measured RMS charge follows from a "U" shape.

The AQS proposed "U" shape for $\mathrm{H}-3$ and $\mathrm{He}-3$ are similar. Both $\mathrm{H}-3$ and He-3 have nine alternating up and down quarks. The "U" structures follow from an arrangment of the nine quarks of each around the vertices of a dodecagon

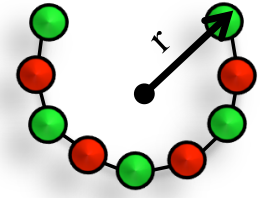

$\mathrm{H}-3$ (12-gon) leaving 3 vertices unoccupied. The difference is that $\mathrm{H}-3$ begins and ends with a down quark, while $\mathrm{He}-3$ begins and ends with an up quark.

The alternation of quarks affects the average center of mass. The two down quarks at the ends of $\mathrm{H}-3$ versus the two up quarks at the ends of $\mathrm{He}-3$ significantly alter the $(x, y)$ coordinates of the respective centers of mass $(\mathrm{cm})$ according to the center of the respective centers of mass $(\mathrm{cm})$ according to the center of mass formula (2). (The z-component is not required as the proposed structures are planar.)

iThe average distance between nucleons of $0.898 \mathrm{fm}$ (SD 0.0585) assumes the charge distribution has a finite surface thickness, yielding a better "goodness-of-fit" than the assuming a uniform charge distribution yielding average distance $0.954 \mathrm{fm}$ (SD 0.13). ${ }^{13}$ 


\begin{tabular}{|c|c|c|c|c|c|c|c|c|}
\hline \multicolumn{9}{|c|}{ Structural Models and Predicted Radii of Light Nuclei (fm) } \\
\hline $\begin{array}{l}\text { Light Nuclides } \\
\text { (measured radius) }\end{array}$ & $\begin{array}{l}\text { H-1 } \\
(.841)\end{array}$ & $\begin{array}{l}\text { H-2 } \\
(2.13)\end{array}$ & $\begin{array}{c}\text { H-3 } \\
(1.76)\end{array}$ & $\begin{array}{l}\mathrm{He-3} \\
(1.97)\end{array}$ & $\begin{array}{c}\mathrm{He}-4 \\
(1.68)\end{array}$ & $\begin{array}{l}\text { Lí-6 } \\
(2.59)\end{array}$ & $\begin{array}{c}\mathbf{L i}-7 \\
(2.44)\end{array}$ & $\begin{array}{l}\text { Average \% } \\
\text { agreement }\end{array}$ \\
\hline $\begin{array}{c}\text { Spherical } \\
\text { Nucleus } \\
\text { (\% agreement with } \\
\text { measured radius) }\end{array}$ & $\begin{array}{l}1.25 \\
(149 \%)\end{array}$ & $\begin{array}{l}1.57 \\
(74 \%)\end{array}$ & $\begin{array}{l}1.80 \\
(102 \%)\end{array}$ & $\begin{array}{l}1.80 \\
(91 \%)\end{array}$ & $\begin{array}{c}1.98 \\
(118 \%)\end{array}$ & $2.27(88 \%)$ & 2.39 (98\%) & $\begin{array}{l}103 \% \\
\text { (SD 24\%) }\end{array}$ \\
\hline $\begin{array}{l}\text { Close-Packed } \\
\text { Nucleons } \\
(\% \text { agreement }) \\
=\text { proton } \\
=\text { neutron }\end{array}$ & $\begin{array}{c}0.841 \\
(100 \%)\end{array}$ & $\begin{array}{l}1.68 \\
(79 \%)\end{array}$ & $\begin{array}{c}1.82 \\
(103 \%)\end{array}$ & $\begin{array}{c}1.82 \\
(92 \%)\end{array}$ & $\begin{array}{c}1.87 \\
(111 \%)\end{array}$ & $\begin{array}{c}2.30 \\
(89 \%)\end{array}$ & $\begin{array}{l}2.27 \\
(93 \%)\end{array}$ & $\begin{array}{c}95 \% \\
\text { (SD 11\%) }\end{array}$ \\
\hline $\begin{array}{l}\text { Alternating } \\
\text { Quarks } \\
\text { (\% agreement) } \\
\text { O= up quark } \\
\text { O=down quark }\end{array}$ & $\begin{array}{c}8 \\
8 \\
0.841 \\
(100 \%)\end{array}$ & $\begin{array}{c}\$ \\
\$ \\
\$ \\
8 \\
2.10 \\
(99 \%)\end{array}$ & $\begin{array}{c}\&_{000^{\circ}} \\
1.88 \\
(107 \%)\end{array}$ & $\begin{array}{c}1.97 \\
(100 \%)\end{array}$ & $\begin{array}{c}1.63 \\
(97 \%)\end{array}$ & $\begin{array}{l}2.42 \\
(93 \%)\end{array}$ & $\begin{array}{l}2.49 \\
(102 \%)\end{array}$ & $\begin{array}{l}99 \% \\
\text { (SD 4\%) }\end{array}$ \\
\hline
\end{tabular}

Figure 3. A representation of the atomic nucleus as an alternating quark sequence produces better average percent agreement and lower standard deviation (SD) with experimental radii of light nuclei in comparison to representational models that assume the nucleus is a sphere sans substructure, or a dense amalgam of close-packed protons and neutrons.

The centers of mass of $\mathrm{H}-3$ and $\mathrm{He}-3$ are not the same because the down quark is nearly twice the mass of the up quark: A down quark a mass of $m_{\mathbf{d}}=4.67 \mathrm{MeV}$ and an up quark has a mass $m_{\mathbf{u}}=2.16 \mathrm{MeV}$. (These quark masses are estimates and may vary as much as 20\%.) ${ }^{12}$ This mass difference results in a slight shift in the centers of mass, which in turn results in differing radius predictions for $\mathrm{H}-3$ and He-3. The AQS calculated radius of $\mathrm{H}-3$ is $\mathrm{r}=1.875 \mathrm{fm}(107 \%$ agreement), and for He-3 $\mathrm{r}=1.969 \mathrm{fm}(100 \%$ agreement).

Finally, for the sake of completeness, a structure for the neutron is included. This structure is similar to the sequential arrangement of quarks within a proton, but positions an up quark between two down quarks. This structure is consistent with experimental charge density results that indicate the neutron has a positive core and negative skin. ${ }^{14,15}$

\section{- STATISTICAL ANALYSIS}

The purpose of statistical analysis is to quantify how well the alternating quark sequence model fits with experimentally determined nuclear radii, and to rule out the possibility that AQS model predictions are a result of coincidence or chance.

Figure 3 compares the percent agreement between representational models and measured RMS charge radii of the light nuclei of interest to nuclear fusion. Of the three representational models, AQS demonstrates the highest average \% agreement with measured RMS charge radii $(99.3 \%$ with a standard deviation is $4 \%$ ). A weaker assumption is that the nucleus comprises close-packed protons and neutrons (average percent agreement $95 \%$ with a standard deviation of $11 \%$ ).
The weakest structural assumption is that of a completely spherical nucleus with no substructure (average percent agreement of $103 \%$ with a standard deviation of $24 \%$ ).

Percent agreement is a useful method of comparing a proposed structure for an individual nuclide to the experimental value, and the average percent agreement is helpful in comparing the three representational models. Correlation coefficients, however, are a preferred way to compare sets of paired data as they include a measure of statistical significance as shown in Table 1.

Table 1. Statistical Correlation Between Measured and Predicted Light Nuclear Radii through Li-7 for Three Representational Nuclear Models.

\begin{tabular}{cccc}
\hline & Pearson's & $\begin{array}{c}\text { Spearman's } \\
\mathbf{r}\end{array}$ & $\begin{array}{c}\text { Kendall's } \\
\mathbf{T}\end{array}$ \\
\hline $\begin{array}{c}\text { Alternating } \\
\text { Quarks }\end{array}$ & $\begin{array}{c}.99 \\
(\mathrm{p}<.001)\end{array}$ & $\begin{array}{c}.96 \\
(\mathrm{p}<.001)\end{array}$ & $\begin{array}{c}.90 \\
(\mathrm{p}=.003)\end{array}$ \\
\hline $\begin{array}{c}\text { Close- } \\
\text { Packed Nu- } \\
\text { cleons }\end{array}$ & $\begin{array}{c}.93 \\
(\mathrm{p}=.002)\end{array}$ & $\begin{array}{c}.67 \\
(\mathrm{p}=.10)\end{array}$ & $\begin{array}{c}.49 \\
(\mathrm{p}=.13)\end{array}$ \\
\hline $\begin{array}{c}\text { Single } \\
\begin{array}{c}\text { Sphere Nu- } \\
\text { cleus }\end{array}\end{array}$ & $\begin{array}{c}.82 \\
(\mathrm{p}=.02)\end{array}$ & $\begin{array}{c}.63 \\
(\mathrm{p}=.13)\end{array}$ & $\begin{array}{c}.39 \\
(\mathrm{p}=.22)\end{array}$ \\
\hline
\end{tabular}

Of the three representational nuclear models, AQS best correlates with the experimentally determined RMS charge radii of the first seven stable light nuclei. Pearson's $r$, Spearman's $\rho$, and Kendall's $T$ are three different methods of determining correlation coefficients, which indicate how well two sets of paired data correlate. AQS coefficients are .90 or 
greater, indicating very strong to near perfect correlation, and $\mathrm{p}$-values $<.01$ indicate the highest level of statistical significance. The radius predictions generated by the other models did not rise to the level of statistical significance by Spearman's $\rho$ and Kendall's T.

\section{- AQS CORRECTIONS}

A central assumption of the alternating quark sequence model is that quark sequences assume simple geometric shapes, and the best fit between the AQS model and accepted radii occurs when this assumption is true. However, the proposed AQS models of $\mathrm{H}-3$, He-4, and Li-6 may deviate from regular polyhedron structures, as the agreement in each of these three cases is greater than $1 \%$. While statistical correlation is strong without correction as shown above, small corrections may nonetheless tweak the AQS predictions more in line with the experimental.

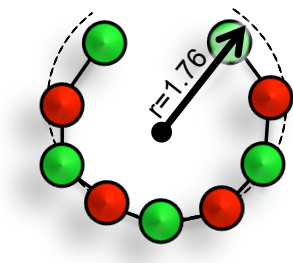

Corrected $\mathrm{H}-3$
The assumed regular polyhedron for $\mathrm{H}-3$ a regular dodecagon having vertex angles equal to $150^{\circ}$. The quarks occupy 9 of 12 vertices to form an open-ended "U" shape as illustrated. The AQS predicted radius of $1.875 \mathrm{fm}$ overestimates the accepted radius of $1.76 \mathrm{fm}$ by $>6 \%$. Narrowing the vertex angles from $150^{\circ}$ to $145.4^{\circ}$ for the 9 occupied vertices, however, brings the predicted radius into $100 \%$ compliance with the accepted $\mathrm{H}-3$ radius of $1.76 \mathrm{fm}$.

The statistical correlations determined above are predicated upon circular structures for He-4, Li-6, and Li-7 that correspond to perfect regular polyhedra. The difference between predicted and measured radii, however, may indicate more of an oval shape for each of these structures.

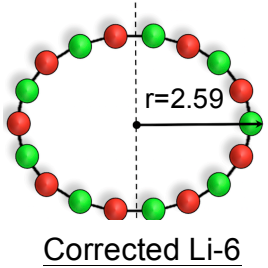

For example, the AQS predicted radius of Li-6 (2.42 fm) underestimates the measured value of $2.59 \mathrm{fm}$ by $7 \%$. The agreement between predicted and experimental improves by assuming a.) the structure has rotational kinetic energy and b.) a measure of flex in the circular structure that allows the circle to deform into a oval with said rotation. The corresponding ovals would have a slightly greater distance from average center of mass to furthest quark, thus bringing the predicted radius into compliance with the experimental radius.

A similar line of reasoning may be applied to the dodecagon of He-4. AQS predicts a radius of $1.63 \mathrm{fm}$ which underestimates the measured value of $1.68 \mathrm{fm}$ by $3 \%$. Rotation of the He-4 ring might flex the structure into an oval, and likewise bring the AQS predicted radius into compliance with measured RMS charge radii values.

The AQS prediction for the radius of lithium-7 is $2.49 \mathrm{fm}$ which overestimates the experimental value $2.44 \mathrm{fm}$ by about $2 \%$. This value improves by assuming an oval shape for Li-7, but for a different reason than Li-6 or He-4. Assuming a measure of laxity in the ring structures of Li- 6 and He- 4 allows an oval deformity. But in the case of $\mathrm{Li}-7$, the six overlapping quarks along one side of the pentadecagon structure may impart a measure of stiffness. This stiffness would draw the furthest quark nearer the average center of mass thus reducing the AQS prediction from $2.49 \mathrm{fm}$ down to the experimental value of $2.44 \mathrm{fm}$.

It is important to note that the statistical correlations with experimental radii were compiled without the corrections suggested in this section, and that even without correction the correlation coefficients are 0.9 or better on a positive correlation scale of 0 to 1.0 .

\section{- AQS LIMITATIONS}

For the stable elements $\mathrm{H}-1$ through $\mathrm{Li}-7$, the radius to mass number curve is erratic and resembles the Sawtooth Mountains. AQS radius predictions of these light nuclei assume quark arrangements around regular polyhedron structures as discussed previously.

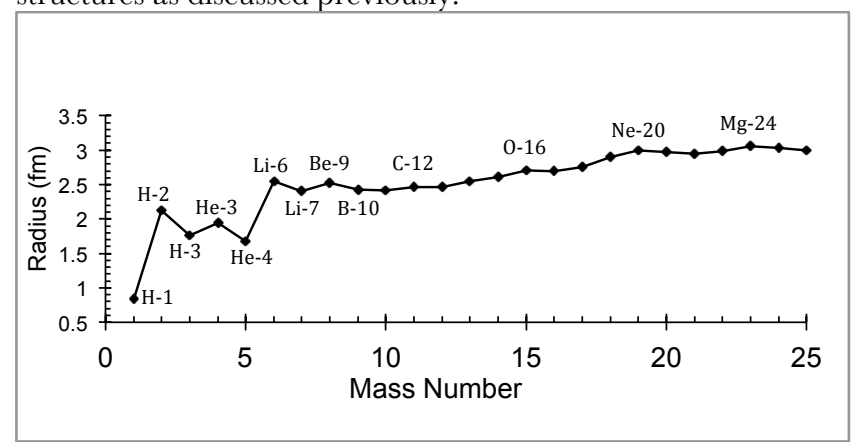

\section{Figure 4. Nuclear radius versus mass number}

For mass numbers above $A=7$, the assumption of a subnucleonic structure comprising an alternating sequence of equally-spaced quarks mays still hold true (Figure 6), but the power of AQS to predict the nuclear radius diminishes as nuclear quark geometries become less regular with increasing mass number.

For nuclear radii above Be-9, the radius to mass number curve is roughly linear, and the radius $r$ is more amenably predicted by the number of nucleons in terms of the formula:

(3) $\quad r=A^{1 / 3} R_{0}$

where $A$ is the mass number and $R_{0}$ is $1.25 \pm 0.2 \mathrm{fm}$. (This formula was used to calculate the radii of the spheres in Table 3.)

A notable exception may be found in the structure of carbon12 , a diamond in the rough. The framework for the 36 quarks of C-12 is a stacked pair of regular 18-gons. The regular polyhedron structure allows an AQS predicted radius of 2.464 fm, representing 99.7\% agreement with the experimental RMS charge radius of $2.4702 \mathrm{fm} .{ }^{10}$ One octadecagon is stacked on top of the other and separated by $0.898 \mathrm{fm}$, consistent with the separation of overlapping strands proposed for the structure of $\mathrm{Li}-7$ above. $\quad(100 \%$ agreement may be achieved by assuming a distance between dodecagon rings of $0.9612 \mathrm{fm})$. Just as in Li-7, alternating quark sequences between the pair of 18-gons associate in such a way that opposite quarks overlap, and protons overlap with neutrons.

\section{- AQS AND THE PROTON PUZZLE}

The "proton puzzle" refers to the differences in proton radius measurements obtained by various investigative teams over the last decade. ${ }^{16}$ Measurements that employ high-energy electron beams yield results $4 \%$ larger than measurements that employ beams of muons. 


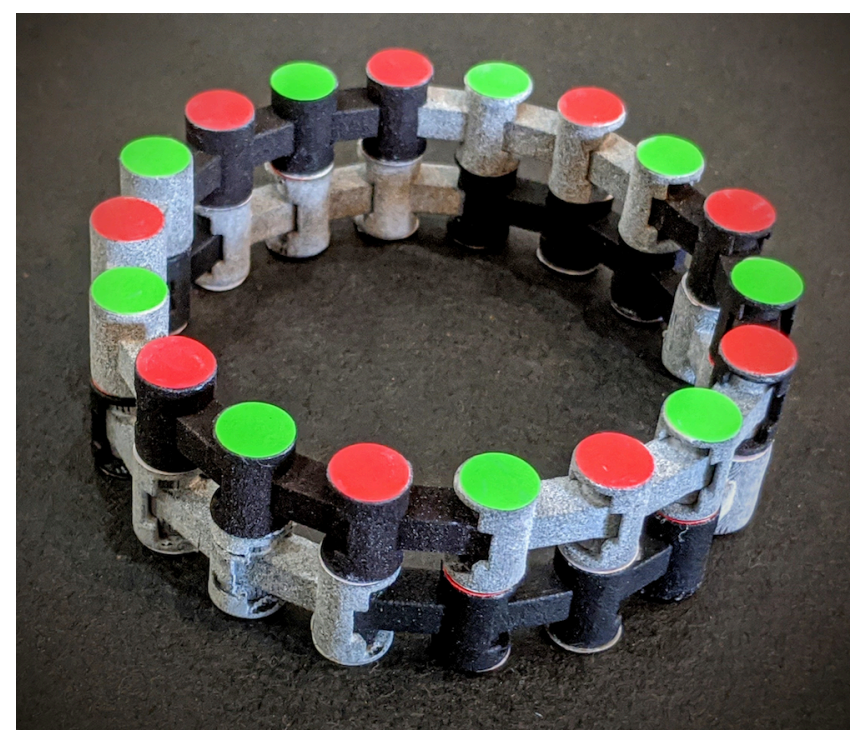

Figure 5 "Ball-and-Stick" scale model of carbon-12. The 36 quarks of C-12 are arranged as a stacked pair of 18-gons. Down quarks (green dots) alternate with up quarks (red dots), and protons (sequence of 3 black links) alternate with neutrons (3 grey links). The 18-gons stack so that protons overlap neutrons.

AQS provides a geometric means of weighing in on the puzzle. While measurements of the proton may vary depending on the type of charged particle beam probe (electron vs. muon), measurements of the helium-4 radius appear largely independent of the beam type. Of the stable light nuclei hydrogen-1 through boron-11, the uncertainty in the measurement of helium- 4 is the smallest at $0.0028 \mathrm{fm}, 10$ even though the reported average radius incorporates both electronic and muonic measurements. ${ }^{18}$ At a time when the radius of the proton has been so uncertain as to be called a puzzle, the measurement of the helium- 4 radius is comparatively certain.

The geometric relationship between helium-4 and proton radii allows a geometric estimate of the size of the proton. As outlined above, the 12 quarks of helium- 4 are arranged as a regular dodecagon with 12 equal sides of length $a$, which equals the radius of the proton. The helium- 4 radius $r$ is related to side $a$ according to formula (1) above. Solving formula (1) for $a$ produces formula (4):

$$
a=2 \mathrm{r} / \csc \left(\frac{\pi}{12}\right)
$$

Substituting the accepted helium- 4 radius $r=1.6755 \pm 0.0028$ $\mathrm{fm}^{8}$ into formula (6) yields a geometric prediction of the proton radius equal to $0.8673 \pm 0.0014 \mathrm{fm}$. (The distance between quarks $a$ is equal to the proton radius in the AQS model). This geometric prediction falls within the range of the last two CODATA recommended values of the proton radius, namely $0.8414 \mathrm{fm}$ from CODATA $2018^{9}$ and $0.8751 \mathrm{fm}$ from CODATA 2014.17

\section{- AQS BALL AND STICK MODELS}

The fusion reaction is shown in nearly every freshman chemistry text as the uniting of two small clusters such as $\mathrm{H}-2$ and $\mathrm{H}-$ 3 to yield a larger cluster $\mathrm{H}-4$, a neutron, and energy. ${ }^{8}$ This depiction persists even though the quark is the most elemental unit of the nucleus, not the proton or neutron.
The same fusion reaction may be depicted in AQS (Figure 7). The intermediary illustrates how H-2 and H-3 might combine in such a way as to form alternating sequences of quarks in He-4 and a neutron. This is very similar to how molecular model kits are used to illustrate simple organic chemistry reactions.

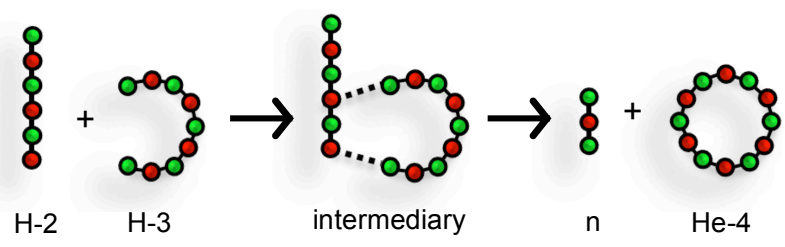

Figure 7. AQS depiction of the nuclear fusion of $\mathbf{H}-2$ and $\mathrm{H}-3$.

Nuclear structures and reactions may be demonstrated with 3D-printed models. These models were designed as an

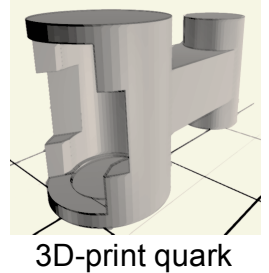
updated ball-and-stick, representing the relative positions of quarks consistent with the parameters of AQS. 3D-printed quark pieces snap together to maintain a fixed quark-to-quark distance of $2 \mathrm{~cm}$, as shown by the model of carbon-12 in Figure 5. The quark design prohibits flexure less than $150^{\circ}$, the vertex angle of the 12-gon of He-4. The .stl files (3-D print files) are available for free download on tinkercad, and nylon is the material of choice. ${ }^{19}$

\section{- AQS AND THE UNCERTAINTY PRINCIPLE}

The Alternating Sequence of Quarks model is a ball-and-stick representational model wherein the ball represents the center of quark mass and the stick represents the constant distance between linked quarks equivalent to the radius of the proton. Mass is related to energy by the formula:

(4)

$$
E=m c^{2}
$$

Since mass and energy are equivalent, the center of quark mass is also the center of quark energy.

This has important implications vis a vis the uncertainty principle, which is generally expressed in terms of an uncertainty in momentum and position:

$$
\Delta x \cdot \Delta p \geq \frac{h}{2 \pi}
$$

The uncertainty principle may, however, be expressed in an alternate form in terms of an uncertainty in total energy and time: ${ }^{20}$

$$
\Delta \boldsymbol{E}_{t} \cdot \Delta \boldsymbol{t} \geq \frac{\boldsymbol{h}}{2 \pi}
$$

Total energy is the sum of kinetic energy (energy of motion) and potential energy (stored energy):

$$
\boldsymbol{E}_{\boldsymbol{t}}=\boldsymbol{E}_{\boldsymbol{k}}+\boldsymbol{E}_{\boldsymbol{p}}
$$

Since the average centers of quark mass in the AQS model are fixed about an average position, the implication of formulas (4) through (7) is that quark energy within the AQS model may be predominantly potential energy, or have a component of potential energy within the context of a classical harmonic oscillator.

Muslulmanbekov proposes such a localized quark/harmonic oscillator within the strongly correlated quark model. SCQM explores potential and kinetic energy components that may allow quark-antiquark pairs to function as 
classic harmonic oscillators about an average position. Similar to AQS, SCQM advances the idea that quark-quark interactions are responsible for nucleon-nucleon binding. SCQM does not appear to include systematic correlation of quark numbers with light nuclear radii, however, which is the main goal of AQS.

\section{- CONCLUSION}

The Alternating Quark Sequence model of the atomic nucleus represents a statistically significant correlation with measured RMS charge radii of light nuclei H-1 through Li-7 according to the parameters listed in Table 2. The geometric and average center of mass calculations are readily verified within a couple of hours with a calculator. Preliminary verification is easy with online calculators of center of mass, regular polyhedra, and correlation coefficients.

AQS models represent the relative positions of quarks but not the forces that maintain these positions. In this way, AQS is similar to the molecular models that van Hoffmann presented decades before the discovery of the electron, proton, and neutron, ${ }^{1}$ and well before Pauling's quantum mechanical wave equations would justify the forces maintaining atoms within molecules. ${ }^{4}$ Watson and Crick demonstrated the power of atomic positional information in the elucidation of the structure of DNA. ${ }^{2}$

The power of the AQS model to predict the nuclear radii of light nuclei is strongest when the constituent quarks take on a regular geometric shape. For heavier nuclei, the parameters outlined in Table 2 may still apply, but geometric irregularity challenges radius predictions. As structures increase in atomic mass number, the irregular shapes may warrant consideration of axis of rotation as well. Preliminary results indicate that structures for nuclei above mass number 7 may be constructed around an octadecagon base of 18 quarks, as foreshadowed by the structure of C-12 in Figure 5 above.

The process of nucleosynthesis may involve the step-wise addition of individual nucleons to a lithium- 6 kernal of 18 quarks. The goal of the next manuscript will be to show how this model of nucleosynthesis produces the relative linearity of the mass number/nuclear radius curve above Be-9 in Figure 4.

Table 2. AQS Parameters.

\section{AQS Parameters}

I. Quarks are linked sequentially.

II. The distance between adjacent linked quarks equals the radius of the proton.

III. The limit of flexure of any 3-quark sequence is approximately equal to $150^{\circ}$, the vertex angle of the dodecagon of helium-4.

IV. Up quarks alternate with down quarks within a quark sequence.

V. Nucleon binding occurs via quark-quark interactions to maintain a continuous sequence of alternating and equally spaced quarks.

VI. Parallel quark sequences may indirectly link or overlap so that protons overlap with neutrons.

VII. Quark sequences tend to form regular polyhedron ring structures where the length of side $a$ equals to the proton radius, and number of sides $n$ equals the quark number.
The role of AQS may be akin to the role of Lewis dot structures, a simple representational model that provides useful but limited information. ${ }^{3}$ Although the current treatment is limited to light nuclei, AQS may have application to outstanding questions regarding the structures of medium and heavy nuclei. AQS may inform the relationship between subnucleonic structure and other nuclear properties, including charge density, magnetic moment, the nucleon pairing phenomenon, the structural role of neutrons in stabilizing heavier nuclei, and the strong nuclear force. Theories of alpha and beta decay may also benefit from from the limited structural information provided by the AQS model.

\section{AUTHOR INFORMATION}

*Raymond J. Walsh

Dr Ray Walsh, PLLC

3556 N Lena Ave

Boise, Idaho 83429

ray.walsh@yahoo.com

\section{ACKNOWLEDGMENTS}

A hardy thanks to Dr Hadley Reed for his steady moral and technical support, thanks to Bill Cheman for his insightful recommendations as to the scope of content, thanks to Patrick McCormick, Laura Cromwell, Brian A. Ertz, and my son Christopher K. Walsh for reviewing the manuscript and providing feedback on readability. Thanks to Earl Swift for his contributions to style and composition.

\section{REFERENCES}

1. Ollis, W. D. "Models and Molecules". Proceedings of the Royal Institution of Great Britain. 45: 1-31. (1972).

2. Watson, J. D. The Double Helix: A Personal Account of the Discovery of the Structure of DNA. (1968) Atheneum.

3. Lewis, G. N. The Atom and the Molecule. J. Am. Chem. Soc. 191638 (4), 762-785.

4. Pauling, L. The Nature of the Chemical Bond. Application of Results Obtained from the Quantum Mechanics and from a Theory of Paramagnetic Susceptibility to the Structure of Molecules. J. Am. Chem. Soc. 1931, 53(4), 1367-1400.

5. da G. Andrade, E. N. Rutherford and the Nature of the Atom (1964).

6. Cook, N. Models of the Atomic Nucleus, 2nd edition, page 48.

7. Ritter, S.K. Cold Fusion Died 25 Years Ago, But The Research Lives On. C\&EN. Nov.7, 2016.

8. Flowers, P., Theopold, K. Chemistry LibreTexts, Chapter 21.8: Nuclear Fusion http://cnx.org/contents/85abf1932bd...a7ac8df6@9.110].

9. D. Newell, K. Pachuki. CODATA recommended values of the fundamental physical constants: 2018 .

10. Angeli, I. \& Marinova, K. P. Table of experimental nuclear ground state charge radii: An update. At. Data Nucl. Data Tables 99, 69 (2013). 
11. J. S. McCarthy, I. Sick, and R. R. Whitney. Electromagnetic structure of the helium isotopes. Phys. Rev. C 15, 1396 - Published 1 April 1977.

12. P.A. Zyla et al. (Particle Data Group), Prog. Theor. Exp. Phys. 2020, 083C01 (2020).

13. Angeli, I. 5. Radius Formulae. International Nuclear Data Committee, Table of Root Mean Square Radii, page 16. 1999.

14. N. Cook. Models of the Atomic Nucleus, 2nd edition, page 131.

15. Weitfeldt, F.E., et al. Measuring the Neutrons' Mean Square Charge Radius Using Neutron Interferometry. arXiv:nucle-ex/0509018v1. 14Sep2005.

16. J. Karr, D. Marchand. Progress on the proton-radius puzzle. Nature. November 6, 2019.

17. P. J. Mohr et al, CODATA recommended values of the fundamental physical constants: 2014, Rev. Mod. Phys. 88, 035009 (2016).
18. Angeli, I. International Nuclear Data Committee, Table of Root Mean Square Radii, Appendix II, page 2. 1999.

19. 3D print quark model stl file may be downloaded at the tinkercad website by typing "Your Rayness quark model" in the search bar.

20. L. I. Mandelshtam, I. E. Tamm. The Uncertainty Relation Between Energy and Time in NonRelativistic Quantum Mechanics. Journal of Physics (USSR). Vol. IX, No. 4. 1945.

21. Musulmanbekov, G. From quark and nucleon correlations to discrete symmetry and clustering in nuclei. arXiv.org > nucl-th > arXiv:1708.04437v2.

Table of Contents graphic:

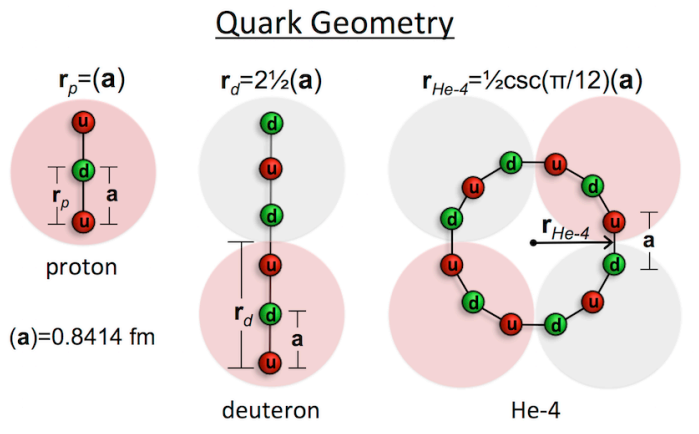

Hero image:

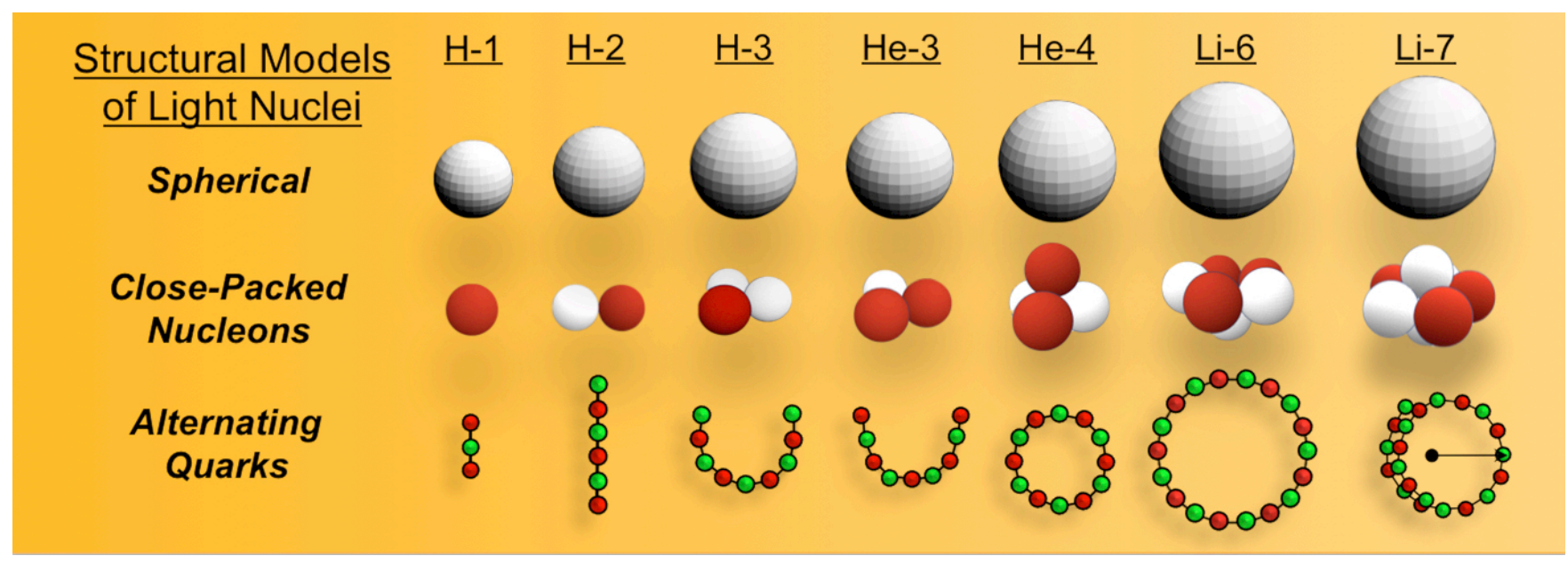

